\title{
Estudo de caso sobre o reforço de estrutura de concreto com fibra de carbono
}

\section{Case study of concrete structures reinforcement with carbon fiber}

\author{
Vitor Hugo Braga ${ }^{\text {1; }}$ Angelo Rubens Migliore Junior ${ }^{2}$ \\ ${ }^{1}$ Graduando do curso de Engenharia Civil do Centro Universitário da Fundação Educacional de Barretos \\ ${ }^{2}$ Professor Doutor do curso de Engenharia Civil do Centro Universitário da Fundação Educacional de \\ Barretos
}

\section{Resumo}

Este trabalho apresenta os resultados experimentais da aplicação de fibra de carbono com objetivo de reforçar estruturas de concreto essencialmente comprimidas, tais como pilares. Esse reforço ocorre pelo confinamento da peça por causa da limitação de deformação transversal em decorrência do efeito de Poisson. Foram moldados corpos de prova cilíndricos de concreto convencional e de concreto reforçado com uma e duas camadas de fibra de carbono. Foi observado aumento de até $48 \%$ na resistência à compressão do exemplar quando reforçado com duas camadas de fibra de carbono. Foram constatados resultados de falhas provenientes de irregularidades na colagem do tecido, não uniformidade e alinhamento das fibras, bem como importância de mão de obra especializada para a execução do reforço, o que pode ser crucial em uma situação real de grande escala.

Palavras-Chave: Estruturas de concreto, Reforço, Fibra de carbono.

\begin{abstract}
This work presents the experimental results of carbon fiber application with the purpose of strengthening compressed concrete structures such as column. The reinforcement occurs due to confinement of the element, because of limitation of the transverse deformation as a result of Poisson's effect. Proof bodies of conventional concrete and reinforced concrete with one and two layers of carbon fiber sheet were molded. An improvement up to $48 \%$ in the compression resistance of the elements reinforced with two layers of carbon fiber was observed. Results of failure in sheet's glue process, the non-uniformity and alignment of the fiber were also observed., such as the necessity of a specialized manpower to perform a well-done reinforcement, which might be crucial in a real large-scale situation.
\end{abstract}

Keywords: Concrete Structures; Reinforcement; Carbon fiber.

\footnotetext{
${ }^{1}$ vitorh.braga@hotmail.com; $+55017981598807^{2}$ rubens.migliore@ $@$ unifeb.edu.br +55017982226732

Recebido em: 01/06/2020

Aceito para a publicação em: 17/06/2020
}

https://doi.org/10.4322/1980-0029.082020 


\section{Introdução}

A recuperação ou o reforço de uma estrutura de concreto já existente para resistir a ações maiores que as previstas em projeto, para corrigir perda de capacidade resistente por causa da deterioração ou para corrigir deficiências relacionadas ao projeto ou à execução têm sido possível por meio do uso de propriedades peculiares de determinados materiais e utilizando técnicas construtivas específicas. Placas de aço coladas externamente, encamisamento por chapas de aço ou novas camadas de concreto e protensão externa são algumas das técnicas atualmente disponíveis (American Concrete Institute, 2008).

Como alternativa para reparo ou reforço estrutural, estão sendo utilizados também materiais compostos de fibras embutidas em resina polimérica, conhecidos como polímeros reforçados com fibra. O uso de fibras de carbono tem apresentado resultados satisfatórios na Construção Civil em razão da elevada resistência mecânica (quando comparado ao concreto), imunidade à corrosão e extrema leveza por causa do baixo peso específico do sistema (cerca de cinco vezes menor que o aço). Essas características destacam o reforço por polímeros com fibra de carbono (PRFC) em relação às demais técnicas, podendo utilizar em diversas geometrias e elementos fletidos de uma estrutura de concreto (International Federation for Structural Concrete, 2001).

Pilares de concreto são elementos essencialmente comprimidos axialmente e podem atingir a ruína quando não apresentam condições de resistir aos esforços a que estão ou estarão submetidos. Os sistemas compostos de camadas de fibras de carbono não são eficientes para a absorção de esforços de compressão. No entanto, impedindo a deformação transversal do concreto comprimido por meio do confinamento da seção de peças, é possível aumentar a resistência e propiciar o aumento na ductibilidade do elemento estrutural reforçado.

Ao aplicar uma força axial de compressão em um corpo deformável, ele se encurta na direção longitudinal e aumenta de dimensão na direção transversal, tal como ilustrado na Figura 1.

Encamisamento é o envolvimento contínuo da peça comprimida de concreto por uma "camisa" composta de camadas com fibras de carbono, conforme ilustra a Figura 2. Segundo Machado (2010), quando a compressão axial atua, a expansão lateral da peça é reduzida pela "camisa" externa, introduzindo uma pressão de fora para dentro do confinamento e criando um estado triplo de tensões de compressão. O confinamento traz como resultado o incremento da resistência à compressão do concreto, fazendo com que o elemento comprimido passe a resistir a esforços antes não suportados.

\section{Materiais e Métodos}

Para a realização deste trabalho, foram utilizados os seguintes materiais:

- Concreto usinado: consumo total de $0,5 \mathrm{~m}^{3} \mathrm{de}$ concreto de classe C30 para a confecção de 18 corpos de prova cilíndricos com diâmetros padronizados de $10 \mathrm{~cm}$ e altura de $20 \mathrm{~cm}$ de concreto produzido em usina local na cidade de Olímpia-SP.

- Tecido de fibra de carbono: consumo total de $1 \mathrm{~m}^{2}$ de tecido de fibra de carbono para reforço estrutural tipo SIKAWRAP 300 C, conforme ilustrado na Figura 3.

- Adesivo para reforço estrutural: adesivo à base de epóxi, de alta viscosidade, composto de uma base (Componente $\mathrm{A}$ ) e um endurecedor (Componente B), de acordo com a Figura 4.

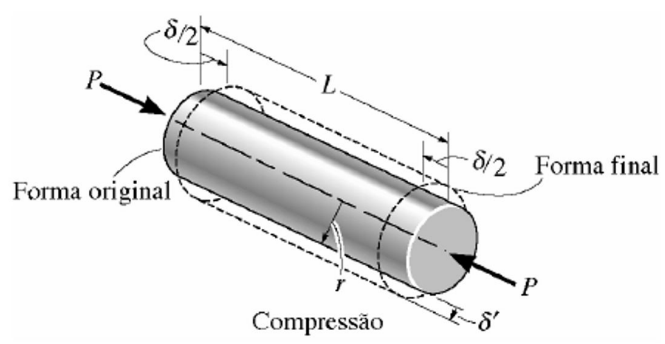

Figura 1. Deformação de um corpo submetido à compressão (Rodrigues, 2010).

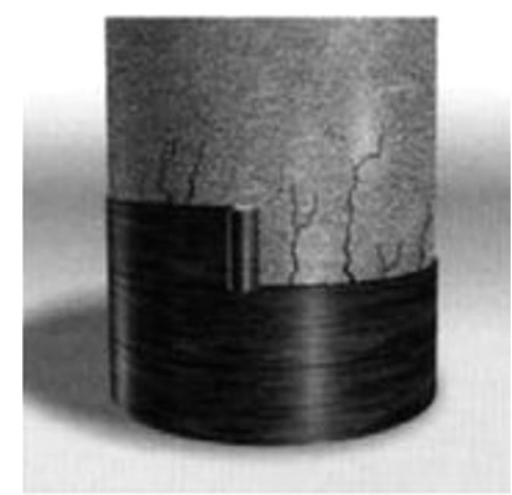

Figura 2. Confinamento de um elemento circular de concreto (Machado, 2010). 


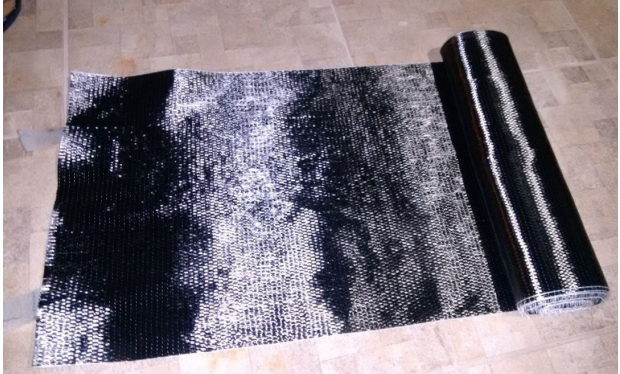

Figura 3. Tecido de fibra de carbono para reforço estrutural (SIKAWRAP 300C).

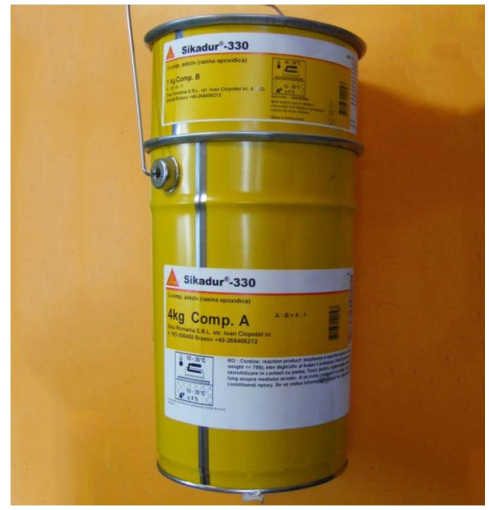

Figura 4. Adesivo epóxi (SIKADUR 330).

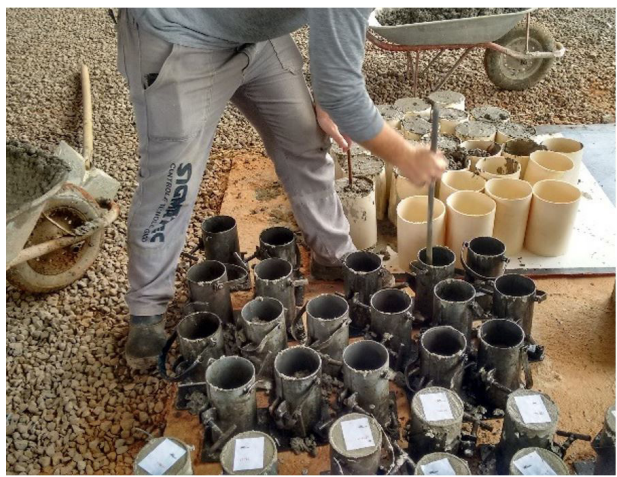

Figura 5. Moldagem de corpos de prova cilíndricos.

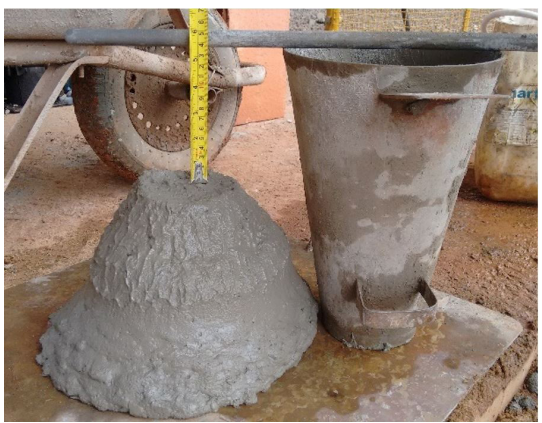

Figura 6. Medida do ensaio de abatimento.
- Prensa hidráulica: para o rompimento dos corpos de prova, foi utilizada prensa de compressão para concreto com capacidade de $2.000 \mathrm{kN}$, pertencente ao laboratório de controle tecnológico Sigmatec, localizado em São José do Rio Preto-SP.

Os corpos de prova foram moldados por profissional de empresa responsável por controle tecnológico no dia 27/03/18, por meio do uso de concreto usinado proveniente de um único caminhão betoneira, o qual foi empregado em concretagem da superestrutura de edifício comercial em obra no município de Olímpia-SP.

A partir da informação do fornecedor do concreto usinado utilizado neste trabalho, a composição do traço do concreto fez uso dos seguintes insumos:

- Cimento CPII-E40: este cimento possui adição de outros materiais em sua mistura, que conferem um menor calor de hidratação, ou seja, menos calor é liberado quando em contato com água. Ele foi fornecido pela empresa Cimento Nacional, com consumo de $370 \mathrm{~kg}$ por $\mathrm{m}^{3}$ de concreto produzido.

- Água: potável proveniente da captação subterrânea, com consumo de 200 litros de água por $\mathrm{m}^{3}$ de concreto.

- Aditivo Polifuncional Maximent NT 14: aditivo plastificante responsável por diminuir o fator água/cimento e melhorar a trabalhabilidade, com consumo de $2,60 \mathrm{~kg}$ por $\mathrm{m}^{3}$ de concreto.

- Areia média: agregado miúdo livre de impurezas com granulometria adequada e estabilidade volumétrica, com consumo de $811 \mathrm{~kg}$ por $\mathrm{m}^{3}$ de concreto.

- Brita 1: agregado graúdo livre de impurezas com granulação uniforme fornecido pela empresa Coplan, com consumo de $1.048 \mathrm{~kg}$ de brita por $\mathrm{m}^{3}$ de concreto.

Os corpos de prova foram moldados conforme parâmetros normativos estabelecidos na norma ABNT NBR 5738:2003 (Associação Brasileira de Normas Técnicas, 2003), sendo realizado em duas camadas com aplicação de 15 golpes em cada uma, visando a uma melhor compactação e retirada de vazios. $\mathrm{O}$ abatimento do concreto utilizado foi de $14 \mathrm{~cm}$. Na Figura 5 é apresentado um detalhe de produção dos corpos de prova, e, na Figura 6, pode ser observado o resultado visual do ensaio de abatimento do concreto utilizado. 
No dia seguinte à concretagem, os corpos de prova foram colocados em bombona plástica e submersos em água portável para realização da cura úmida, conforme a Figura 7, permanecendo ali pelo período de 21 dias.

Após o período de cura, ou seja, no dia $17 / 04 / 18$, os corpos de prova foram retirados da água, e o sistema de reforço com fibra de carbono foi aplicado em 10 peças. Primeiramente, os corpos de prova foram limpos com uma estopa banhada em álcool para a remoção de possíveis impurezas. O material responsável pela aderência entre o substrato e o tecido foi adesivo estrutural à base de epóxi bicomponente, sendo a base " $\mathrm{A}$ " e o endurecedor " $B$ " (equivalência 4:1 em massa respectivamente), e aplicado com a utilização de espátula de aço. A Figura 8 mostra uma porção de adesivo já misturado, e a Figura 9 ilustra a aplicação no corpo de prova a ser ensaiado.

$\mathrm{O}$ tecido de fibra de carbono foi recortado em faixas de $35 \mathrm{~cm}$ x $20 \mathrm{~cm}$ e aplicados no sentido de envolver lateralmente a peça cilíndrica, conforme mostram as Figuras 10 e 11. Após 60 minutos da aplicação, as amostras com duas camadas de fibra de carbono receberam novamente a aplicação do adesivo estrutural à base de epóxi e outra camada de tecido de fibra de carbono.

Após completar 28 dias após a confecção, ou seja, no dia 24/04/18, todos os corpos de prova foram submetidos a ensaio de compressão axial, realizado na empresa Sigmatec, localizada no município de São José do Rio Preto-SP, e conforme parâmetros estabelecidos na norma ABNT NBR 5739:1994 (Associação Brasileira de Normas Técnicas, 1994). Para regularização das faces inferior e superior de cada corpo de prova, foi utilizada retífica, e, para o ensaio, foi feito uso da prensa hidráulica EMIC com capacidade de $2.000 \mathrm{kN}$. A prensa hidráulica possui painel digital em que as cargas máximas suportadas são apresentadas, tal como ilustra a Figura 12.

\section{Resultados}

Os resultados de ensaios de compressão dos 18 corpos de prova (carga de ruptura e tensão de ruptura) estão apresentados na Tabela 1.

Esses dados foram organizados no gráfico apresentado na Figura 13, em cuja legenda consta o concreto convencional correspondente aos corpos de prova que não foram reforçados, e os demais resultados se referem aos corpos de prova reforçados com uma ou duas camadas de fibra de carbono.

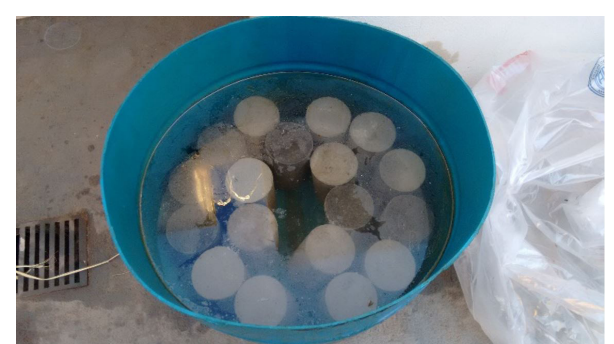

Figura 7. Tanque com corpos de prova para cura em água.

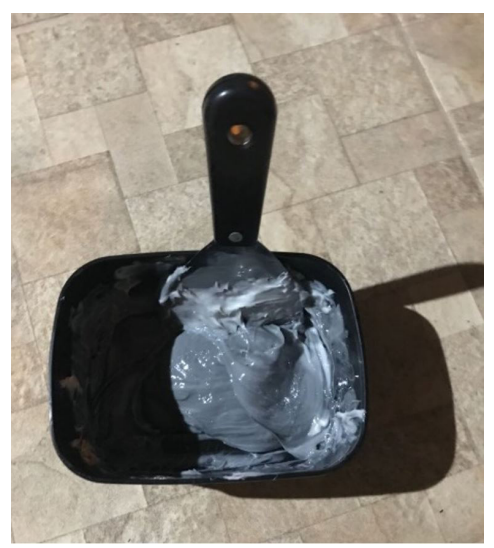

Figura 8. Aspecto de mistura de adesivo estrutural à base de epóxi.

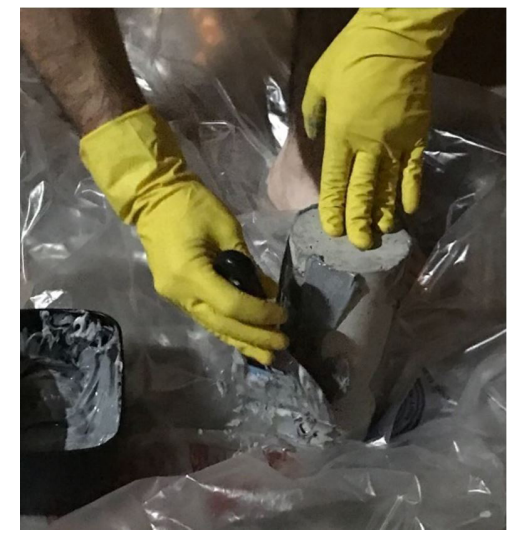

Figura 9. Aplicação de adesivo estrutural.

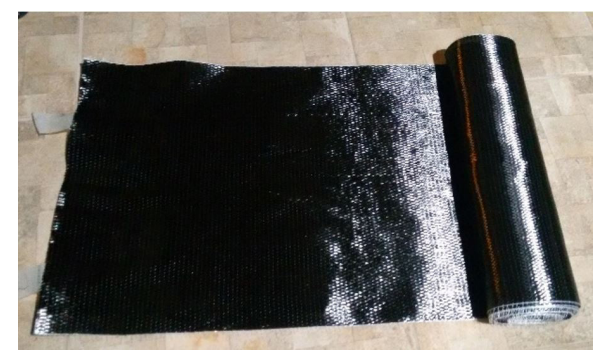

Figura 10. Tecido de fibra de carbono. 


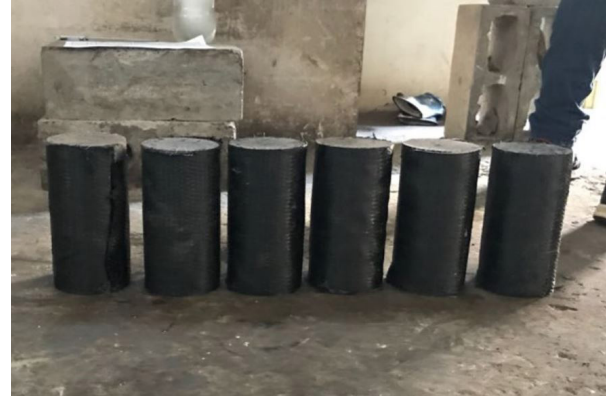

Figura 11. Amostras reforçadas com fibra de carbono.

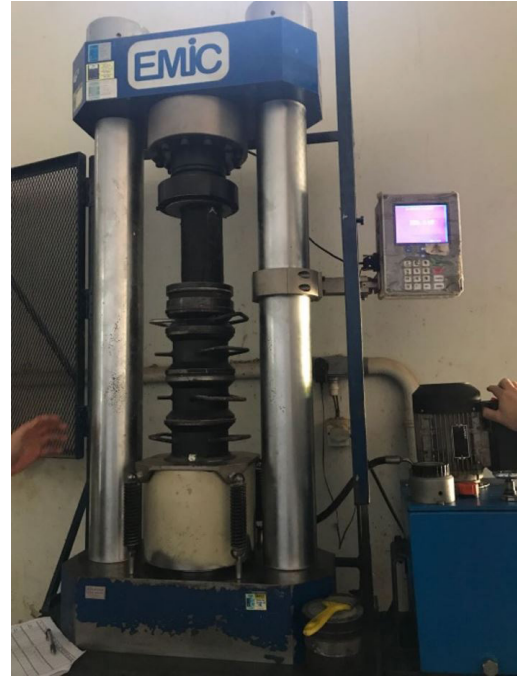

Figura 12. Prensa hidráulica EMIC.
Foi também realizado estudo estatístico para a verificar a confiabilidade dos dados obtidos, bem como encontrar desvios e variações nos resultados. A Tabela 2 mostra a análise dos resultados tendo como base o equacionamento apresentado por Bessegato (2004).

Sendo:

- Média $[\bar{X}]$ : soma das tensões dividida pelo número de amostras.

$\bar{X}=\frac{\sum \text { Tensão }}{n^{o} \text { amostras }}$

- Variância [Var]: soma dos quadrados dos desvios dividida pelo número de amostras.

$\operatorname{Var}=\frac{\sum\left(X_{i}-\bar{X}\right)^{2}}{n^{o} \text { amostras }}$

- Desvio-padrão [DP]: módulo da raiz quadrada da variância.

$D P=|\sqrt{\operatorname{Var}}|$

- Coeficiente de variação [CV]: razão do desvio-padrão pela média.

$C V=\frac{D P}{\bar{X}}$

- Margem de erro [E]: variação aceitável do resultado medido a partir da média.

$$
E=1,645 * \frac{D P}{\sqrt{N}}
$$

Tabela 1. Geometria, carga e tensão de ruptura dos corpos de prova.

\begin{tabular}{|c|c|c|c|c|c|}
\hline Tipo & $\mathbf{C P}$ & Diâmetro (mm) & Área $\left(\mathrm{cm}^{2}\right)$ & Ruptura (kN) & Tensão (MPa) \\
\hline \multirow{8}{*}{$\begin{array}{l}\text { Concreto } \\
\text { convencional }\end{array}$} & 1 & 100 & 78,60 & 313,60 & 39,90 \\
\hline & 2 & 100 & 78,57 & 318,20 & 40,50 \\
\hline & 3 & 100 & 78,55 & 313,43 & 39,90 \\
\hline & 4 & 100 & 78,58 & 335,54 & 42,70 \\
\hline & 5 & 100 & 78,62 & 310,54 & 39,50 \\
\hline & 6 & 100 & 78,63 & 320,82 & 40,80 \\
\hline & 7 & 99 & 76,22 & 323,94 & 42,50 \\
\hline & 8 & 102 & 81,07 & 334,01 & 41,20 \\
\hline \multirow{6}{*}{$\begin{array}{l}\text { Reforço (1 } \\
\text { camada) }\end{array}$} & 1 & 100 & 79,17 & 348,96 & 44,08 \\
\hline & 2 & 100 & 79,06 & 394,75 & 49,93 \\
\hline & 3 & 101 & 79,33 & 370,94 & 46,76 \\
\hline & 4 & 100 & 79,02 & 360,07 & 45,56 \\
\hline & 5 & 100 & 79,07 & 358,72 & 45,37 \\
\hline & 6 & 100 & 79,16 & 374,06 & 47,25 \\
\hline \multirow{4}{*}{$\begin{array}{l}\text { Reforço (2 } \\
\text { camadas) }\end{array}$} & 1 & 101 & 79,42 & 564,02 & 71,02 \\
\hline & 2 & 101 & 79,44 & 495,02 & 62,31 \\
\hline & 3 & 101 & 79,47 & 429,94 & 54,10 \\
\hline & 4 & 101 & 79,47 & 428,37 & 53,91 \\
\hline
\end{tabular}




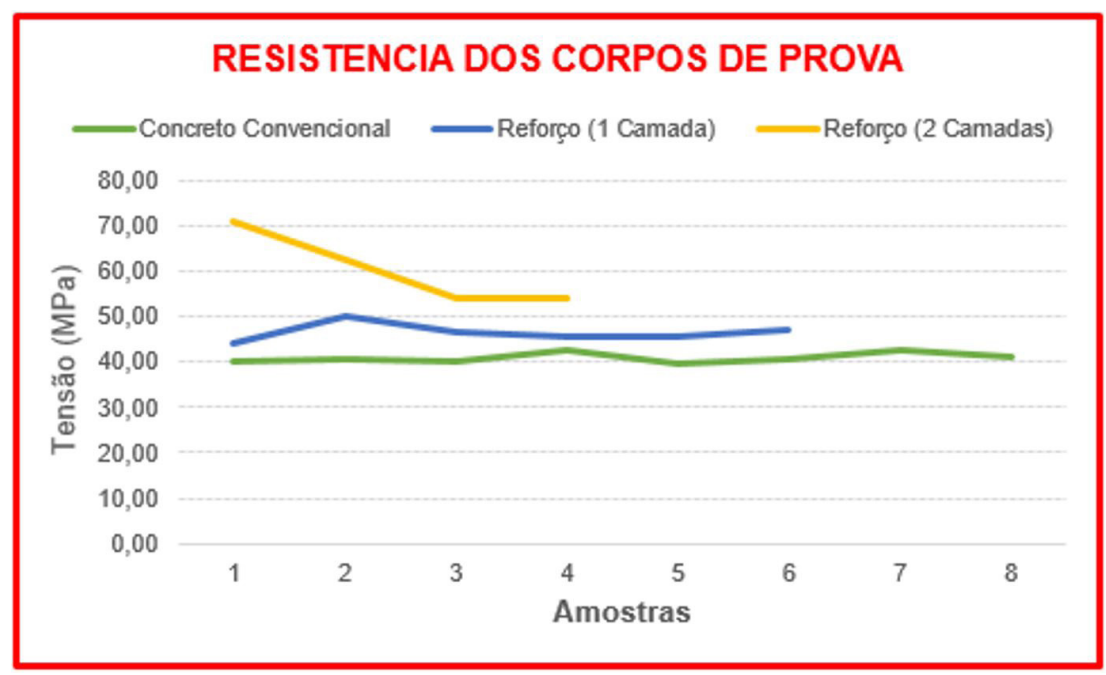

Figura 13. Gráfico de resistências dos corpos de prova, segundo o tipo de reforço.

Tabela 2. Estudo estatístico dos resultados obtidos.

\begin{tabular}{ccccccc}
\hline Dados estatísticos & Média & Variância & Desvio-padrão & $\begin{array}{c}\text { Coeficiente } \\
\text { de variação }\end{array}$ & $\begin{array}{c}\text { Margem } \\
\text { de erro }\end{array}$ & $\begin{array}{c}\text { Margem de } \\
\text { confiança }\end{array}$ \\
\hline Concreto convencional & 40,88 & 1,25 & 1,12 & 0,03 & 0,65 & $40,22<\mu<41,53$ \\
Reforço (1 camada) & 46,49 & 3,41 & 1,85 & 0,04 & 1,24 & $45,25<\mu<47,73$ \\
Reforço (2 camadas) & 60,33 & 49,54 & 7,04 & 0,12 & 5,79 & $54,54<\mu<66,12$ \\
\hline
\end{tabular}

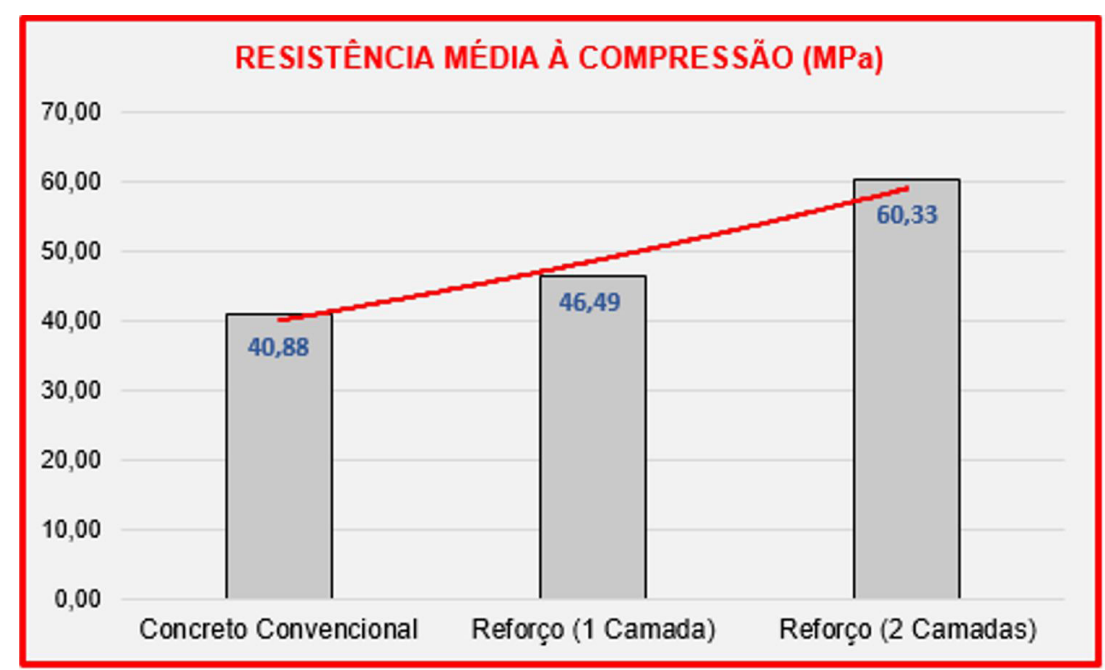

Figura 14. Resistência média à compressão.

\section{Discussão de resultados}

O gráfico da Figura 14 mostra que o reforço com uso de duas camadas de fibra de carbono trouxe maior eficiência em comparação ao reforço com uma camada, pois a resistência média à compressão das amostras aumentou de $46,49 \mathrm{MPa}$ para 60,33 MPa.
É perceptível o aumento na resistência à compressão de corpos de prova cilíndricos de concreto simples utilizados neste estudo. A partir das médias dos valores obtidos, podem ser encontradas as seguintes relações entre as resistências à compressão:

$$
f_{f 1}=1,137 \times f_{c 0} \text { e } f_{f 2}=1,476 \times f_{c 0}
$$




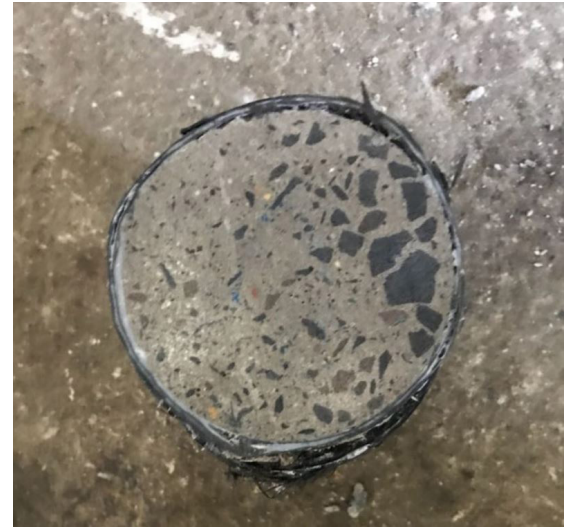

Figura 15. Falha na colagem do tecido.

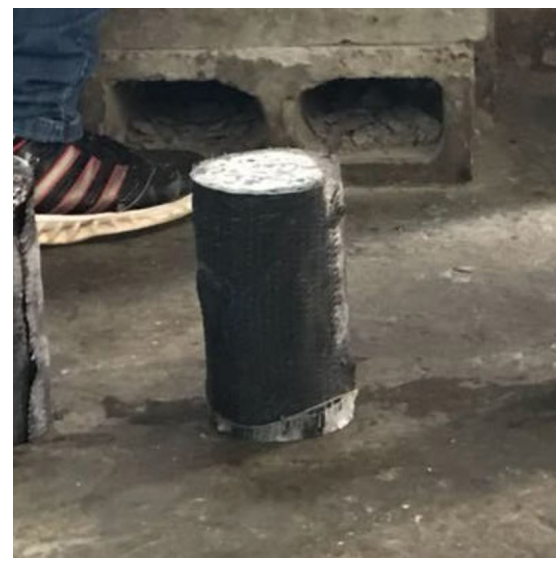

Figura 16. Desalinhamento das fibras de reforço.

Sendo:

- $\mathrm{f}_{\mathrm{c} 0}=$ resistência média de amostras de concreto convencional.

- $\mathrm{f}_{\mathrm{fl}}=$ resistência média de amostras reforçadas com uma camada de fibra de carbono.

- $\mathrm{f}_{\mathrm{f} 2}=$ resistência média de amostras reforçadas com duas camadas de fibra de carbono.

Foi constatado aumento de resistência quando comparado o resultado das amostras com uma camada de fibra de carbono (14\%) ao das amostras com duas camadas de fibra de carbono (48\%) em relação ao concreto convencional. Segundo Carrazedo (2005), quanto maior o número de camadas de fibras de carbono no reforço, maior será a resistência do elemento estrutural, entretanto um aumento exagerado de camadas não resultará em um aumento da resistência na mesma proporção, reafirmado por Santos et al. (2013, p.48): “[...] a eficiência diminui de acordo com o acréscimo de camadas."
As amostras reforçadas com duas camadas de fibra de carbono apresentaram maior variação de resistência, pois os valores de tensões mínimas e máximas foram de 53,91 MPa e 71,02 MPa, respectivamente. Foram detectadas falhas de execução com relação à colagem da segunda camada de tecido de fibra de carbono, como mostram as Figuras 15 e 16. Segundo Machado (2010), desalinhamento das fibras ou aderência ineficiente podem ocasionar redução na resistência efetiva do sistema composto com PRFC (Polímero de fibra de carbono reforçado).

Neste estudo, não foi possível garantir a eficiência na aplicação das fibras por causa da inexperiência da mão de obra ao aplicar o reforço, visto que ele feito pelo autor deste RF (Reforço com fibras).

\section{Conclusões}

A eficiência do reforço com a utilização de PRFC pode ser comprovada por meio do aumento na resistência das amostras ensaiadas. Foi observado que o aumento de resistência não é proporcional ao aumento do número de camadas de fibra de carbono, sendo recomendada, por questões de viabilidade e de economia, a utilização do mínimo de camadas necessárias para atender à resistência desejada.

Pode ser comprovada também a necessidade de mão de obra especializada para a aplicação do reforço, visto que resultados mais expressivos poderiam ser obtidos em situações em que há disponibilidade de mão de obra própria para a execução.

\section{Agradecimentos}

Os autores agradecem às empresas e pessoas que forneceram alguma forma de ajuda para a realização deste estudo, a saber: Rio Verde Engenharia, pelo suporte na fabricação e transporte das amostras; Pires Giovanetti Guardia, pelo fornecimento de SIKAWRAP e SIKADUR 300; Flexmix Concreto, pelo fornecimento do concreto; Sigmatec Controle Tecnológico, pela moldagem e rompimento das amostras.

\section{Referências bibliográficas}

American Concrete Institute - ACI. (2008). ACI 440.2R-08: Guide for the design and construction of externally bonded FRP systems for strengthening concrete structures. Farmington Hills: ACI. 
Associação Brasileira de Normas Técnicas ABNT. (1994). NBR 5739: Concreto - Ensaio de compressão de corpos de prova cilíndricos. Rio de Janeiro: ABNT.

Associação Brasileira de Normas Técnicas - ABNT. (2003). NBR 5738: Concreto - Procedimento para moldagem e cura de corpos de prova. Rio de Janeiro: ABNT.

Bessegato, L. F. (2004). Estatística aplicada. Belo Horizonte: Pontifícia Universidade Católica de Minas Gerais.

Carrazedo, R. (2005). Mecanismos de confinamento em pilares de concreto encamisados com polímeros reforçados com fibras submetidos à flexo-compressão (Tese de doutorado). São Carlos: Universidade de São Paulo.
International Federation for Structural Concrete FIB. (2001). Bulletin 14: Externally bonded FRP reinforcement for $R C$ structures. Switzerland: FIB.

Machado, A. P. (2010). Manual de reforço das estruturas de concreto armado com fibras de carbono. São Paulo.

Rodrigues, L. E. M. J. (2010). Deformações e propriedades mecânicas dos materiais (Nota de aula). São Paulo: Instituto Federal de Educação, Ciência e Tecnologia.

Santos, G. M. S., Ross, H. L. S., \& Habitzreuter, L. (2013). Análise da eficiência do reforço estrutural com fibra de carbono em pilares curtos (Trabalho de conclusão de curso). Universidade Tecnológica Federal do Paraná, Curitiba. 